\title{
Microbial Susceptibility Test
}

National Cancer Institute

\section{Source}

National Cancer Institute. Microbial Susceptibility Test. NCI Thesaurus. Code C85540.

A method to determine whether microbial growth is affected by an antimicrobial compound. 